\title{
The Association between Pathologies of Voice and Gastro-Oesophageal Reflux Disease (GORD)*
}

\author{
Rasan Burhan" ${ }^{1 \#, ~ J a l a l ~ M o r a d z a d e h ², ~ E t h a n ~ S m a l l w o o d ~}{ }^{3}$, Thomas Kiwanuka4, Samuel Ajayi ${ }^{5}$ \\ ${ }^{1}$ St Georges University Hospitals NHS Foundation Trust, London, United Kingdom \\ ${ }^{2}$ Luton and Dunstable NHS Trust, Luton, United Kingdom \\ ${ }^{3}$ Nottingham University Hospitals NHS Trust, Nottingham, United Kingdom \\ ${ }^{4}$ Brighton and Sussex University Hospitals NHS Trust, Brighton, United Kingdom \\ ${ }^{5}$ Silversands Pharmacy, London, United Kingdom \\ Email: "rasan@doctors.net.uk, jalal.moradzadeh@ldh.nhs.uk, mzyers@nottingham.ac.uk, Thomas.kiwanuka1@nhs.net, \\ Samuel.ajayi@nhs.net
}

How to cite this paper: Burhan, R., Moradzadeh, J., Smallwood, E., Kiwanuka, T. and Ajayi, S. (2020) The Association between Pathologies of Voice and GastroOesophageal Reflux Disease (GORD). Open Access Library Journal, 7: e6890. https://doi.org/10.4236/oalib.1106890

Received: October 11, 2020

Accepted: November 8, 2020

Published: November 11, 2020

Copyright $\odot 2020$ by author(s) and Open Access Library Inc.

This work is licensed under the Creative Commons Attribution International License (CC BY 4.0).

http://creativecommons.org/licenses/by/4.0/

(c) (i) Open Access

\begin{abstract}
Voice and speaking are integral parts of a person's self-identity and ability to communicate on a day-to-day basis. There have been numerous studies investigating the link between gastro-esophageal reflux disease and its concomitant association with a range of other disorders across the breadth of medicine. In this review, we will focus on the relationship between conditions which contribute to pathologies of voice and GORD. These will include Laryngopharyngeal reflux, Globus Pharyngeus and Paroxysmal Laryngospasm. To perform this review, we scoped papers from journals in Gastroenterology, Otolaryngology and General Medicine across the medical literature focusing on PubMed indexed articles. Inclusion criteria included English as the primary language of the work and peer-reviewed journals. We scoped a range of papers from 1988 to the current decade. More than 40 original research and review papers-in addition to guidelines and books-were utilised to supplement the review. The key terms outlined related to "Gastro-oesophageal reflux disease", "Laryngopharyngeal reflux", "Globus Pharyngeus", "Paroxysmal Laryngospasm" as well as their respective abbreviations were used in the initial scope of the search. We found that there was a link between Gastro-oesophageal reflux disease and pathologies of voice due to the intercurrent relationship with pathologies which contribute to the changes in voice aforementioned. Investigating and implementing effective treatments for gastro-oesophageal reflux disease and related disorders can help individuals to regain their natural voice-an attribute closely linked to their sense of self-resulting in a profoundly positive psychological impact.
\end{abstract}




\section{Subject Areas}

Gastroenterology \& Hepatology, Otorhinolaryngology, Pathology

\section{Keywords}

Voice Pathology, Gastro-Esophageal Reflux Disease, Laryngopharyngeal Reflux, Globus Pharyngeus, Paroxysmal Laryngospasm

\section{Introduction}

Gastro-oesophageal reflux disease is a condition where the contents of the stomach pass back upwards into the oesophagus rather than passing through towards and into the small intestine, where it continues to undergo the process of digestion. Most people, at some points in their lives, experience gastro-oesophageal reflux disease (to differing degrees), however, it is only considered to be a problem when it causes noticeable or significant symptoms or complications arising as a result [1]. Its prevalence in the general population has increased in recent times, causing an impairment in the quality of life for sufferers. It has been suggested that gastro-oesophageal reflux disease is the costliest gastrointestinal disease in terms of healthcare expenditure [2]. It is, in essence, a multifactorial disease dependent on a number of genetic and environmental factors. It has been suggested that it has a correlation with the increasing prevalence of obesity and dietary factors [3]. This is further supported by studies showing its association with a high Body Mass Index (value $>25 \mathrm{~kg} / \mathrm{m}^{2}$ ) [4]. Studies have also indicated that there is a negative correlation between $H$. pylori infection and gastro-oesophageal reflux disease; its eradication has been associated with the development of gastro-oesophageal reflux disease [5].

The disease manifests itself through a range of symptoms. Sufferers frequently complain of heartburn, a sensation of burning in the chest that occurs after a meal. This can cause a lot of anxiety in patients who mistake it for symptoms of a heart attack or acute angina. Acid reflux is also a common symptom which is caused by the stomach acid regurgitating back into the oral cavity. This can lead to further problems, such as dental disease and erosion due to the demineralising effects of a low pH [6]. Problems with swallowing are also commonplace. These include odynophagia and dysphagia, pain and difficulty in swallowing. This can result in a loss of appetite and subsequent weight loss [7]. By looking at some of the pathologies associated with GORD, which are often linked to voice pathologies, we can begin to appreciate the indirect relationship that exists. The purpose of this review is to look into some of these associated pathologies to further elucidate how they may bear impact on an individual's voice.

\section{Methods}

To perform this review, we scoped papers from journals in Gastroenterology, 
Otolaryngology and General Medicine from across the medical literature focusing on PubMed indexed articles. Inclusion criteria included English as the primary language of the work and peer-reviewed journals. We scoped a range of papers from 1988 to the current decade. More than 40 original research and review papers-in addition to guidelines and books-were utilized to supplement the review. The key terms outlined related to "Gastro-oesophageal reflux disease", "Laryngopharyngeal reflux", "Globus Pharyngeus", "Paroxysmal Laryngospasm" as well as their respective abbreviations were used in the initial scope of the search. We also looked into secondary references from those obtained in the main journals relating to this topic-to further research some of the main findings and their relevance to the project.

\section{Results}

A proportion of those with Gastro-oesophageal reflux disease go on to develop Barrett's oesophagus [3]. Normally, the epithelium of the oesophagus is squamous up to the lower oesophageal sphincter. In Barrett's, the epithelium becomes columnar which can lead to dysplasia. It is often asymptomatic however can present with gastrointestinal symptoms such as heartburn, nausea and vomiting, hence the need for endoscopies for individuals with Gastro-oesophageal reflux disease and Barrett's [8] [9] [10]. Studies have shown that there is a 10-fold increased risk of oesophageal cancer with Barrett's. Despite this, there is only a slightly increased risk of oesophageal cancer in patients with reflux without Barrett's [11].

In patients with a pathological reflux, it has been found that there are three main underlying mechanisms. Firstly, there is a transitory complete relaxation of the lower oesophageal sphincter. Secondly, the resistance of the anti-reflux barrier is overcome due to a transient increase in the intra-abdominal pressure. Thirdly, reflux occurs through a permanently hypotonic sphincter. It has also been suggested that diaphragmatic contraction may assist in preventing reflux resulting from an increase in intra-abdominal pressure.

With relation to voice pathology, it is worthwhile to consider laryngopharyngeal reflux. This is a condition where the gastric contents reflux back into the laryngopharynx. This anatomical structure extends from the superior border of the epiglottis through to the inferior border of the cricoid cartilage. It manifests itself through a sore throat and cough alongside symptom's relating to pathology of voice: namely; hoarseness (difficulty making sounds when speaking), dysphonia (difficulty speaking) and globus (a sensation of a lump or foreign body in the throat). There is evidence to suggest that there is a significant prevalence of laryngopharyngeal symptoms in more than half of patients with Gastro-oesophageal reflux disease [12]. Other studies have indicated that there may be a link between suffering from gastro-oesophageal reflux disease and developing laryngeal cancer [13].

The actual mechanism leading to the symptoms present in this disorder are currently still being researched. However, it has been suggested that the acidic 
nature of gastric juice may be responsible for causing some tissue damage in the upper airways [14]. Over the past half a decade, it has been suggested that pepsin and bile salts are damaging influences on Proton Pump Inhibitor therapy and they may in actual fact contribute to its negative effects. It has been speculated that the reason for this could be related to the model of receptor-mediated endocytosis of pepsin by laryngeal epithelial cells. Proton Pump Inhibitors are not very efficient as whilst they reduce acid exposure, they do not have a direct effect on pepsin secretion, hence reflux will not be prevented [15].

The diagnosis of Laryngopharyngeal reflux is also an area of great debate. ENT specialists commonly diagnose it using laryngoscopy (where edema and erythema are present) [16]. The specificity of this approach however has come under question since there has been a study which has shown that signs of laryngeal irritation are evident in over $80 \%$ of asymptomatic and healthy controls [17].

One approach utilised is the Reflux Symptom Index (RSI). In this, there are 9 distinct questions each relating to a set of symptoms, based upon patients experience in the past month. Areas explored include hoarseness, clearing throat, postnasal drip and difficulties in swallowing amongst a range of other symptoms related to the condition. Each symptom is given a score depending of the severity of each problem. This has also been used to monitor and assess changes in the quality of life experienced by patients after treatment has been started [18]. It is interesting to note that knowledge and insight into the Reflux Symptom Index and Laryngopharyngeal reflux is lacking amongst primary care physicians in some countries [19].

There are several approaches to treating Laryngopharyngeal reflux. One major approach is through the use of lifestyle modifications and education. This approach was adopted in district hospital in the United Kingdom within the past decade and had been shown to be an effective management solution [15]. This method involves advising patients to reduce daily caloric intake, increase aerobic physical activity, increase fruit vegetable and fiber intake whilst reducing carbohydrate and sweet food intake. One practical solution mentioned refers to changing the position of the bed to elevate the head; reducing reflux due to the effect of gravity [20]. Obesity, as previously mentioned, has both an impact on Gastro-oesophageal reflux disease and its inter-related condition Laryngopharyngeal reflux. This is because obesity is believed to modify the oesophago-gastric joint in terms of morphology and function. It does this by causing a mechanical disruption of the joint by separating the lower oesophageal sphincter and the crural diaphragm. The reflux symptoms themselves improve significantly with a decrease in weight [21]. With regards to diet, there has been conflicting evidence as to whether a high dietary fat intake is correlated with an increased risk of the disorder since it has been shown that this could be the case, although other studies carried out in the past have suggested otherwise [22] [23] [24]. Fibre has been shown in various studies to reduce symptoms of Ga- 
stro-oesophageal reflux disease significantly (suggested to be about $50 \%$ reduction) [25]. The mechanism for this is unknown as of yet, however there have been suggestions that its consumption could lead to increased gastric emptying-this would tie in well with the mechanisms through which the disease manifests itself [20].

As aforementioned, it has been found that elevating the head of a bed can decrease oesophageal acid exposure hence leading to a quicker clearance of the oesophagus alongside a lessened reflux period [26]. In addition to this, there is also evidence to suggest that falling asleep in the right lateral recumbent position can extend the reflux time and hence it would be advisable to avoid this position [27].

Perhaps one of the most effective non-medical interventions in helping to manage the condition involves incorporating aerobic physical exercise (of at least 30 minutes) into a patient's routine. Studies have shown that individuals with Gastro-oesophageal reflux disease tend to be less physically active in comparison to those without the condition [28]. Hence, it's likely that encouraging exercise in affected individuals would lead to better management of symptoms. Paradoxically, it has been found that intense bouts of exercise and activity increase oesophageal acid. This occurs since an increased frequency of abdominal straining predisposes to subsequent reflux back through the oesophago-gastric junction [29].

Pharmacological management of Laryngopharyngeal reflux is also of paramount importance. It is commonly acknowledged that the use of Proton Pump Inhibitor's is of great utility in treating and managing Gastro-oesophageal reflux disease [30]. However, this may not the case for Laryngopharyngeal reflux where its efficacy remains a doubt and its limitations acknowledged. The reasoning for this lies in the idea that the upper aero-digestive tract has a greater level of sensitivity to acid refluxes as opposed to the oesophagus [20]. As a result, higher doses of Proton Pump Inhibitor's coupled with longer periods of use are initiated in order to elicit improvements in laryngeal symptoms [31]. Given this, its actual benefit is still of great uncertainty and several studies have failed to show any such benefits [32] [33]. It appears that where an improvement has been noted with the use of Proton Pump Inhibitor's, further examination has shown that the improvements were related to symptoms such as heartburn and not those localised to the throat [34]. Hence, given the co-existing nature of the conditions as previously discussed, this further supports the idea that despite such therapy not being effective specifically for Laryngopharyngeal reflux, it does have an overall benefit in those suffering from co-existing Laryngopharyngeal reflux and Gastro-oesophageal reflux disease. This is further supported by a study demonstrating that improvements in laryngeal symptoms were greater in patients with Gastro-oesophageal reflux disease as opposed to those without [35]. It may however be worth noting that for longer term $\mathrm{H} 2$-antagonists may generally be better tolerated in contrast to PPI's with fewer side-effects. 
Liquid alginate suspension has also been studied to decipher its value in the treatment of Laryngopharyngeal reflux. A study carried out in Nottingham found that administering it led to significant improvements in a patient's symptom scores after a few months of use [36]. This is further confirmed by research investigating its anti-reflux properties. It was found that symptoms are likely to improve with its use as the suspension. Mechanisms for this relate to it forming an anti-reflux barrier and limiting the proximal migration of reflux from the oesophagus [37].

Surgical interventions have also been adopted in the treatment of Laryngopharyngeal reflux. Laparoscopic fundoplication in particular has shown great promise. Its safety and efficacy have been shown in clinical trials and it was found that it was beneficial in reducing symptoms in patients [38]. Interestingly, it has been shown that its effectiveness is most evident in patients who respond well to Proton Pump Inhibitor therapy and less so to patients who don't respond well to intensive Proton Pump Inhibitor therapy [39]. The intervention, whilst beneficial, is not without its potential post-operative effects. It has been shown that there is a chance of developing dysphagia, flatulence, diarrhoea and bloating following the operation. Furthermore, the possibility of symptoms recurring exists [40].

Globus Pharyngeus is often a complaint in patients with Laryngopharyngeal reflux. It has shown that speech and language therapy could lead to positive outcomes in addressing this particular symptom. The main principles underlying this relates to relieving laryngopharyngeal tension besides voice exercises [41]. In addition to this, laryngeal therapies and breathing exercises focusing on the diaphragm have also shown promise in this area of therapy [42] [43]. This demonstrates the importance of not relying strictly on pharmacological and surgical interventions where other procedures-like this approach-can have equally positive outcomes.

Paroxysmal laryngospasm is a condition where the vocal folds forcefully adduct for extended periods of time leading to closure of the glottis and potential obstruction of the airways. It's associated with a sensation of choking at the level of the larynx alongside apnea (temporary cessation of breaking) and aphonia (inability to speak). Studies and reports have linked the condition with Gastro-oesophageal reflux disease. As a result, it has been shown to respond well to the previously aforementioned anti-reflux treatments [44].

\section{Conclusions}

Speech is one of the fundamental components of human interaction. As a result, changes in a patient's voice due to disease can have many detrimental effects. Emerging research has linked Gastro-oesophageal reflux disease to voice changes in aspect of voice quality and tone [45]. Such changes would be likely to increase anxiety in social situations and in severe cases lead to withdrawal and fear of speech. Communication with partner's and children would also be of concern. 
This is a big psychological burden. Voice changes in patients with Gastro-oesophageal reflux disease appears to be at least partly reversible following effective treatment and this would also likely lead to improvements in confidence, anxiety and feelings of self-worth. Voice changes are not usually monitored during treatment by healthcare professionals but may prove useful in assessing disease severity, prognosis and improvement in the condition. This is an avenue which could be looked into further.

To conclude, studies and findings from literature have indicated that there is a link between gastro-oesophageal reflux disease and pathologies of voice. This has meant that treatments currently used for Gastro-oesophageal reflux disease have been evaluated for their effectiveness in treating these inter-related disorders, which have an impact on an individual's voice. Today, it is accepted that voice is integral to social communication and an individual's personality [46]. Hence, it can be inferred that investigating and implementing effective treatments will enable individuals to regain an attribute so closely linked to their sense of self, resulting in a profoundly positive psychological impact.

\section{Acknowledgements}

With many thanks to Mr Phillips (ENT Consultant) in Yorkshire for training and overseeing the special training module in the Pathology of Voice at York Hospital during undergraduate medical training.

\section{Funding}

No funding obtained.

\section{Conflicts of Interest}

No conflicts of interest from any of the authors involved in work.

\section{References}

[1] Galmiche, J.P. and Janssens, J. (1995) The Pathophysiology of Gastro-Oesophageal Reflux Disease: An Overview. Scandinavian Journal of Gastroenterology, 211, 7-18. https://doi.org/10.3109/00365529509090286

[2] Voutilainen, M. (2014) Gastro-Oesophageal Reflux Disease-A Common and Benign But Costly Disease. Duodecim, 130, 801-807.

[3] Parasa, S. and Sharma, P. (2013) Complications of Gastro-Oesophageal Reflux Disease. Best Practice \& Research: Clinical Gastroenterology, 27, 433-442. https://doi.org/10.1016/j.bpg.2013.07.002

[4] Dore, M.P., Maragkoudakis, E., Fraley, K., Pedroni, A., Tadeu, V., Realdi, G., et al. (2008) Diet, Lifestyle and Gender in Gastro-Esophageal Reflux Disease. Digestive Diseases and Sciences, 53, 2027-2032. https://doi.org/10.1007/s10620-007-0108-7

[5] Xie, T., Cui, X., Zheng, H., Chen, D., He, L. and Jiang, B. (2013) Meta-Analysis: Eradication of Helicobacter pylori Infection Is Associated with the Development of Endoscopic Gastroesophageal Reflux Disease. European Journal of Gastroenterology \& Hepatology, 25, 1195-1205. https://doi.org/10.1097/MEG.0b013e328363e2c7 
[6] Hellwig, E. and Lussi, A. (2014) Oral Hygiene Products, Medications and Drugs-Hidden Aetiological Factors for Dental Erosion. Monographs in Oral Science, 25, 155-162. https://doi.org/10.1159/000359942

[7] Kahrilas, P.J. (2000) Strategies for Medical Management of Reflux Disease. Best Practice \& Research: Clinical Gastroenterology, 14, 775-791. https://doi.org/10.1053/bega.2000.0124

[8] Jeffrey, S. (2010) Tobias, Cancer and Its Management. 6th Edition, Wiley-Blackwell, Chichester.

[9] National Institute for Health and Care Excellence (NICE) (2004) Dyspepsia: Managing Dyspepsia in Adults in Primary Care.

[10] National Institute for Health and Care Excellence (NICE) (2010) Interventional Procedure Guidance 344: Epithelial Radiofrequency Ablation for Barrett's Oesophagus.

[11] Solaymani-Dodaran, M., Logan, R.F., West, J., Card, T. and Coupland, C. (2004) Risk of Oesophageal Cancer in Barrett's Oesophagus and Gastro-Oesophageal Reflux. Gut, 53, 1070-1074. https://doi.org/10.1136/gut.2003.028076

[12] Jaspersen, D., Kulig, M., Labenz, J., Leodolter, A., Lind, T., Meyer-Sabellek, W., et al. (2003) Prevalence of Extra-Oesophageal Manifestations in Gastro-Oesophageal Reflux Disease: An Analysis Based on the ProGERD Study. Alimentary Pharmacology \& Therapeutics, 17, 1515-1520.

https://doi.org/10.1046/j.1365-2036.2003.01606.x

[13] Vaezi, M.F., Qadeer, M.A., Lopez, R. and Colabianchi, N. (2006) Laryngeal Cancer and Gastroesophageal Reflux Disease: A Case-Control Study. The American Journal of Medicine, 119, 768-776. https://doi.org/10.1016/j.amjmed.2006.01.019

[14] Wiener, G.J., Tsukashima, R., Kelly, C., Wolf, E., Schmeltzer, M., Bankert, C., et al. (2009) Oropharyngeal pH Monitoring for the Detection of Liquid and Aerosolized Supraesophageal Gastric Reflux. Journal of Voice, 23, 498-504.

https://doi.org/10.1016/j.jvoice.2007.12.005

[15] Pearson, J.P., Parikh, S., Orlando, R.C., Johnston, N., Allen, J., Tinling, S.P., et al. (2011) Review Article: Reflux and Its Consequences-The Laryngeal, Pulmonary and Oesophageal Manifestations. Conference Held in Conjunction with the 9th International Symposium on Human Pepsin (ISHP) Kingston-upon-Hull, UK, 21-23 April 2010. Alimentary Pharmacology \& Therapeutics, 33, 1-71. https://doi.org/10.1111/j.1365-2036.2011.04581.x

[16] Vaezi, M.F., Hicks, D.M., Abelson, T.I. and Richter, J.E. (2003) Laryngeal Signs and Symptoms and Gastroesophageal Reflux Disease (GERD): A Critical Assessment of Cause and Effect Association. Clinical Gastroenterology and Hepatology, 1, 333-344. https://doi.org/10.1053/S1542-3565(03)00177-0

[17] Milstein, C.F., Charbel, S., Hicks, D.M., Abelson, T.I., Richter, J.E. and Vaezi, M.F. (2005) Prevalence of Laryngeal Irritation Signs Associated with Reflux in Asymptomatic Volunteers: Impact of Endoscopic Technique (Rigid vs. Flexible Laryngoscope). Laryngoscope, 115, 2256-2261.

https://doi.org/10.1097/01.mlg.0000184325.44968.b1

[18] Lee, J.S., Lee, Y.C., Kim, S.W., Kwon, K.H. and Eun, Y.G. (2014) Changes in the Quality of Life of Patients with Laryngopharyngeal Reflux after Treatment. Journal of Voice: Official Journal of the Voice Foundation, 28, 487-491. https://doi.org/10.1016/j.jvoice.2013.12.015

[19] Chorti, M.S., Prokopakis, E.P., Lahanas, V.A., Bessas, Z., Papadakis, Y., Velegrakis, S.G., et al. (2013) Knowledge of Primary Care Doctors about Laryngopharyngeal 
Reflux Disease. $B-E N T$, 9, 53-56.

[20] Martinucci, I., de Bortoli, N., Savarino, E., Nacci, A., Romeo, S.O., Bellini, M., et al. (2013) Optimal Treatment of Laryngopharyngeal Reflux Disease. Therapeutic Advances in Chronic Disease, 4, 287-301. https://doi.org/10.1177/2040622313503485

[21] Pandolfino, J.E., El-Serag, H.B., Zhang, Q., Shah, N., Ghosh, S.K. and Kahrilas, P.J. (2006) Obesity: A Challenge to Esophagogastric Junction Integrity. Gastroenterolo$g y$, 130, 639-649. https://doi.org/10.1053/j.gastro.2005.12.016

[22] Jacobson, B.C., Somers, S.C., Fuchs, C.S., Kelly, C.P. and Camargo, C.A. (2006) Body-Mass Index and Symptoms of Gastroesophageal Reflux in Women. The New England Journal of Medicine, 354, 2340-2348.

https://doi.org/10.1056/NEJMoa054391

[23] Mangano, M., Colombo, P., Bianchi, P.A. and Penagini, R. (2002) Fat and Esophageal Sensitivity to Acid. Digestive Diseases and Sciences, 47, 657-660. https://doi.org/10.1023/A:1017944925601

[24] Pehl, C., Pfeiffer, A., Waizenhoefer, A., Wendl, B. and Schepp, W. (2001) Effect of Caloric Density of a Meal on Lower Oesophageal Sphincter Motility and Gastro-Oesophageal Reflux in Healthy Subjects. Alimentary Pharmacology \& Therapeutics, 15, 233-239. https://doi.org/10.1046/j.1365-2036.2001.00919.x

[25] Terry, P., Lagergren, J., Ye, W., Wolk, A. and Nyren, O. (2001) Inverse Association between Intake of Cereal Fiber and Risk of Gastric Cardia Cancer. Gastroenterology, 120, 387-391. https://doi.org/10.1053/gast.2001.21171

[26] Hamilton, J.W., Boisen, R.J., Yamamoto, D.T., Wagner, J.L. and Reichelderfer, M. (1988) Sleeping on a Wedge Diminishes Exposure of the Esophagus to Refluxed Acid. Digestive Diseases and Sciences, 33, 518-522. https://doi.org/10.1007/BF01798350

[27] Khoury, R.M., Camacho-Lobato, L., Katz, P.O., Mohiuddin, M.A. and Castell, D.O. (1999) Influence of Spontaneous Sleep Positions on Nighttime Recumbent Reflux in Patients with Gastroesophageal Reflux Disease. The American Journal of Gastroenterology, 94, 2069-2073. https://doi.org/10.1111/j.1572-0241.1999.01279.x

[28] Djarv, T., Wikman, A., Nordenstedt, H., Johar, A., Lagergren, J. and Lagergren, P. (2012) Physical Activity, Obesity and Gastroesophageal Reflux Disease in the General Population. World Journal of Gastroenterology, 18, 3710-3714. https://doi.org/10.3748/wjg.v18.i28.3710

[29] Pandolfino, J.E., Bianchi, L.K., Lee, T.J., Hirano, I. and Kahrilas, P.J. (2004) Esophagogastric Junction Morphology Predicts Susceptibility to Exercise-Induced Reflux. The American Journal of Gastroenterology, 99, 1430-1436. https://doi.org/10.1111/j.1572-0241.2004.30515.x

[30] Galmiche, J.P., Zerbib, F. and des Varannes, S.B. (2013) Treatment of Gastro-Oesophageal Reflux Disease: Three Decades of Progress and Disappointments. United European Gastroenterology Journal, 1, 140-150. https://doi.org/10.1177/2050640613484021

[31] Park, W., Hicks, D.M., Khandwala, F., Richter, J.E., Abelson, T.I., Milstein, C., et al. (2005) Laryngopharyngeal Reflux: Prospective Cohort Study Evaluating Optimal Dose of Proton-Pump Inhibitor Therapy and Pretherapy Predictors of Response. Laryngoscope, 115, 1230-1238. https://doi.org/10.1097/01.MLG.0000163746.81766.45

[32] Vaezi, M.F., Richter, J.E., Stasney, C.R., Spiegel, J.R., Iannuzzi, R.A., Crawley, J.A., et al. (2006) Treatment of Chronic Posterior Laryngitis with Esomeprazole. Laryngoscope, 116, 254-260. https://doi.org/10.1097/01.mlg.0000192173.00498.ba 
[33] El-Serag, H.B., Lee, P., Buchner, A., Inadomi, J.M., Gavin, M. and McCarthy, D.M. (2001) Lansoprazole Treatment of Patients with Chronic Idiopathic Laryngitis: A Placebo-Controlled Trial. The American Journal of Gastroenterology, 96, 979-983. https://doi.org/10.1111/j.1572-0241.2001.03681.x

[34] Lam, P.K., Ng, M.L., Cheung, T.K., Wong, B.Y., Tan, V.P., Fong, D.Y., et al. (2010) Rabeprazole Is Effective in Treating Laryngopharyngeal Reflux in a Randomized Placebo-Controlled Trial. Clinical Gastroenterology and Hepatology, 8, 770-776. https://doi.org/10.1016/j.cgh.2010.03.009

[35] Lien, H.C., Wang, C.C., Liang, W.M., Sung, F.C., Hsu, J.Y., Yeh, H.Z., et al. (2013) Composite $\mathrm{pH}$ Predicts Esomeprazole Response in Laryngopharyngeal Reflux without Typical Reflux Syndrome. Laryngoscope, 123, 1483-1489.

https://doi.org/10.1002/lary.23780

[36] McGlashan, J.A., Johnstone, L.M., Sykes, J., Strugala, V. and Dettmar, P.W. (2009) The Value of a Liquid Alginate Suspension (Gaviscon Advance) in the Management of Laryngopharyngeal Reflux. European Archives of Oto-Rhino-Laryngology, 266, 243-251. https://doi.org/10.1007/s00405-008-0708-7

[37] Zentilin, P., Dulbecco, P., Savarino, E., Parodi, A., Iiritano, E., Bilardi, C., et al. (2005) An Evaluation of the Antireflux Properties of Sodium Alginate by Means of Combined Multichannel Intraluminal Impedance and pH-Metry. Alimentary Pharmacology \& Therapeutics, 21, 29-34. https://doi.org/10.1111/j.1365-2036.2004.02298.x

[38] Sala, E., Salminen, P., Simberg, S., Koskenvuo, J. and Ovaska, J. (2008) Laryngopharyngeal Reflux Disease Treated with Laparoscopic Fundoplication. Digestive Diseases and Sciences, 53, 2397-2404. https://doi.org/10.1007/s10620-007-0169-7

[39] Chen, R.Y. and Thomas, R.J. (2000) Results of Laparoscopic Fundoplication Where Atypical Symptoms Coexist with Oesophageal Reflux. ANZ Journal of Surgery, 70, 840-842. https://doi.org/10.1046/j.1440-1622.2000.01981.x

[40] Richter, J.E. (2013) Gastroesophageal Reflux Disease Treatment: Side Effects and Complications of Fundoplication. Clinical Gastroenterology and Hepatology, 11, 465-471. https://doi.org/10.1016/j.cgh.2012.12.006

[41] Khalil, H.S., Bridger, M.W., Hilton-Pierce, M. and Vincent, J. (2003) The Use of Speech Therapy in the Treatment of Globus Pharyngeus Patients. A Randomised Controlled Trial. Revue de Laryngologie-Otologie-Rhinologie (Bord), 124, 187-190.

[42] Pacheco, A., Cobeta, I. and Wagner, C. (2013) Refractory Chronic Cough: New Perspectives in Diagnosis and Treatment. Archivos de Bronconeumología, 49, 151-157. https://doi.org/10.1016/j.arbres.2012.09.009

[43] Eherer, A.J., Netolitzky, F., Hogenauer, C., Puschnig, G., Hinterleitner, T.A., Scheidl, S., et al. (2012) Positive Effect of Abdominal Breathing Exercise on Gastroesophageal Reflux Disease: A Randomized, Controlled Study. The American Journal of Gastroenterology, 107, 372-378. https://doi.org/10.1038/ajg.2011.420

[44] Bortolotti, M. (1989) Laryngospasm and Reflex Central Apnoea Caused by Aspiration of Refluxed Gastric Content in Adults. Gut, 30, 233-238.

https://doi.org/10.1136/gut.30.2.233

[45] Schneider, G.T., Vaezi, M.F. and Francis, D.O. (2016) Reflux and Voice Disorders: Have We Established Causality? Current Otorhinolaryngology Reports, 4, 157-167. https://doi.org/10.1007/s40136-016-0121-5

[46] Li, J. and Xu, S. (2020) Extraversion, Neuroticism, and Employee Voice: A Conservation of Resources Perspective. Frontiers in Psychology, 11, 1281.

https://doi.org/10.3389/fpsyg.2020.01281 